\title{
IL-6 upregulation contributes to the reduction of miR-26a expression in hepatocellular carcinoma cells
}

\author{
Yafei Zhang ${ }^{1,2}$, Bicheng Zhang ${ }^{1}$, Anran Zhang ${ }^{2}$, Xiaohua Li ${ }^{3}$, Jian Liu ${ }^{1}$, Jie Zhao ${ }^{1}$, Yong Zhao ${ }^{1}$, \\ Jianfei Gao ${ }^{1}$, Dianchun Fang ${ }^{2}$ and Zhiguo Rao ${ }^{1}$ \\ ${ }^{1}$ Department of Oncology, Wuhan General Hospital of Guangzhou Command, People's Liberation Army, Wuhan, China \\ ${ }^{2}$ Department of Gastroenterology, Southwest Hospital, Third Military Medical University, Chongqing, China \\ ${ }^{3}$ State Key Laboratory of Cancer Biology and Xijing Hospital of Digestive Diseases, Fourth Military Medical University, Xi'an, \\ China
}

\begin{abstract}
A recent study showed that miR-26a is downregulated in hepatocellular carcinoma tissues and that this downregulation is an independent predictor of survival. Interestingly, the same study also reported that miR-26a downregulation causes a concomitant elevation of IL-6 expression. Because miR-26a expression was found to be transcriptionally downregulated by oncogene c-Myc in various cancers, and the expression of c-Myc was increased by IL-6 stimulation, we hypothesized that IL-6 contributes to reduction of miR-26a in hepatocellular carcinoma. Serum IL-6 was measured by ELISA and miR-26a was detected by qRT-PCR. The data of 30 patients with hepatocellular carcinoma who had undergone surgical tumor resection revealed that serum IL-6 could be considered to be a predictor of survival up to 5 years for hepatocellular carcinoma patients (log-rank test, $\mathrm{P}<0.05$ ). We observed that the serum IL-6 concentration was inversely correlated with miR-26a expression in cancerous tissues (Pearson correlation test, $r=-0.651, \mathrm{P}<0.01$ ). Furthermore, by in vitro experiments with HepG2 cells, we showed that IL-6 stimulation can lead to miR-26a suppression via c-Myc activation, whereas in normal hepatocyte LO2 cells incubation with IL-6 had no significant effect on miR-26a expression. Taken together, these results indicate that miR-26a reduction in hepatocellular carcinoma might be due to IL-6 upregulation.
\end{abstract}

Key words: MiR-26a; IL-6; c-Myc; Hepatocellular carcinoma; Carcinoma prognosis

\section{Introduction}

MicroRNAs (miRNAs) are endogenous small noncoding RNAs that regulate cellular gene expression and are functionally linked to tumorigenesis (1). While some of the miRNAs are oncogenic, miRNAs such as miR-26a are consistently downregulated in a number of cancers, demonstrating their potential tumor suppressor functions $(2,3)$. Recently, a study by Ji et al. (4) revealed that miR$26 a$ is downregulated in hepatocellular carcinoma (HCC) and that this downregulation is associated with poor survival. Therefore the authors indicated that the reduction of miR-26a expression may be used as an independent predictor of survival for HCC patients. In the cited study, the authors also found that the miR-26a reduction in cancerous tissues is accompanied by a concomitant elevation in interleukin (IL)-6 expression (4). miR-26a expression has been found to be transcriptionally downregulated by oncogene c-Myc in a variety of human cancers $(3,5,6)$. Interestingly, several studies have determined that IL-6 can induce a significant induction of c-Myc expression in cancer cells including human HCC HepG2 cells (7-9). Therefore, it is reasonable to hypothesize that the miR-26a reduction in HCC is the consequence, at least in part, of elevated IL-6 expression.

IL-6 is a multifunctional cytokine that was originally characterized as a regulator of immune and inflammatory responses (10). Recent evidence has revealed that IL-6 also plays a prominent role in the pathogenesis of tumor cells $(11,12)$. Supporting the role of IL-6 in human tumors is the observation of elevated serum concentrations of IL-6 and their association with a poor clinical outcome $(12,13)$. In addition, IL-6 produced by tumor cells also acts as a paracrine or autocrine regulatory factor and therefore regulates tumor cell behaviors including metastasis, proliferation and survival $(12,14)$. The prognostic value

Correspondence: Zhiguo Rao, Department of Oncology, Wuhan General Hospital of Guangzhou Command, People's Liberation Army, Wuhan 430070, China. E-mail: raozhiguo2012@163.com

Received April 6, 2012. Accepted September 12, 2012. First published online September 28, 2012. 
of serum IL-6 has been well demonstrated in various tumors. Furthermore some studies pointed out that the prognostic value of serum IL-6 is independent of other known prognostic variables (15-17). The current study was designed to examine the prognostic value of serum IL-6 regarding survival and the correlation between serum IL-6 and tumor miR-26a expression in HCC patients. Furthermore, we determined whether IL- 6 stimulation can induce the reduction of miR-26a expression via activation of c-Myc in HCC cells.

\section{Material and Methods}

\section{Clinical samples}

Thirty pairs of cancerous tissues and adjacent noncancerous tissues and matched serum samples were collected from 30 patients with early stage HCC (21 cases of stage I and 9 cases of stage II, determined according to criteria of the International Union against Cancer) who underwent surgical tumor resection between 2003 and 2005 at Xijing Hospital (Fourth Military Medical University, Xi'an, China) and Southwest Hospital (Third Military Medical University, Chongqing, China). None of the patients received neoadjuvant chemotherapy or radiation therapy before surgery. The patients with confirmed cirrhosis or acute infectious diseases (detectable serum hepatitis $B$ virus (HBV) DNA or hepatitis $C$ virus RNA, and severe bacterial infection) were excluded from the study. All patients studied were males, with a mean age of 46 years (range $=36-61$ years). Tissue samples were collected from each patient immediately after surgical removal and snap-frozen in liquid nitrogen for subsequent analysis. Serum samples were collected before surgery by low-speed centrifugation of heparinized whole blood and stored in liquid nitrogen until assayed.

\section{Follow-up assessments}

Follow-up assessments were performed at annual intervals for up to 5 years after surgery by phone and mail. The median follow-up time was 49.5 months (range $=9$ to 60 months). Among the 30 patients who participated in this study, 11 patients who survived longer than 5 years without recurrence after curative surgery for $\mathrm{HCC}$ were classified as the no recurrence group, whereas the remaining 19 patients were classified as the recurrence group (survival $<5$ years for recurrence and $>5$ years with recurrence).

\section{Detection of serum IL-6}

The concentration of serum IL- 6 was determined in duplicate using commercially available enzyme-linked immunosorbent assay kits (R\&D Systems, Inc., USA) according to manufacturer instructions.

\section{Detection of miR-26a expression}

Total RNA from frozen tissues or cells was isolated with Trizol (Takara, China) according to manufacturer instructions. The TaqMan stem-loop RT-PCR method was used to assess the expression of mature miR-26a (miR26a-1) with kits from Applied Biosystems (USA) as described previously (18). The real-time PCR results, recorded as threshold cycle numbers, were normalized against an internal control (18S rRNA), and then reported as fold-changes. Primers used for PCR amplification were: 5'-CCGCCGTTCAAGTAATCCAGGA-3' and 5'GTGCAGGGTCCGAGGT-3'.

\section{Ethics statement}

Ethics approval for the research was obtained from the Ethics Committee of Xijing Hospital and Southwest Hospital, and written informed consent was obtained from all patients who participated in the study.

\section{Cell culture and IL-6 stimulation}

Human HCC HepG2 cells and normal hepatocyte LO2 cells were routinely cultured in RPMI 1640 medium (HyClone, USA) supplemented with $10 \%$ fetal bovine serum (HyClone) at $37^{\circ} \mathrm{C}$ in a $5 \% \mathrm{CO}_{2}$ incubator. For stimulation, cells were starved with serum-free medium for $6 \mathrm{~h}$ and then incubated with different doses of human recombinant IL-6 (Sigma-Aldrich, USA) for $2 \mathrm{~h}$, after which total proteins and RNA were extracted. For the blocking assay, HepG2 cells were pre-incubated with $100 \mu \mathrm{M}$ of c-Myc inhibitor 10058-F4 (Sigma-Aldrich) for $6 \mathrm{~h}$ before IL-6 stimulation (19).

\section{Cell transfection}

LO2 cells transfected with plasmids expressing c-Myc (pcDNA3.1-c-Myc, provided by Dr. Xiaohua Li, State Key Laboratory of Cancer Biology and Xijing Hospital of Digestive Diseases, Fourth Military Medical University, Xi'an, China) were selected with medium containing G418 (Sigma-Aldrich) for 6 weeks to generate a stable cell line with higher expression of c-Myc. LO2 cells transfected with blank pcDNA3.1 plasmids were used as control.

\section{Western blot analysis}

Total cell lysates were prepared in 1X SDS buffer. Proteins were separated by SDS-PAGE and transferred to PVDF membranes. Membranes were then blotted with antibodies specific for c-Myc and $\beta$-actin (Sigma-Aldrich). Antigen-antibody complexes were visualized using enhanced chemiluminescence (Sigma-Aldrich).

\section{Statistical analysis}

Data are reported as means \pm SEM. Differences were compared by one-way ANOVA followed by the least significant difference $t$-test. Correlations between two groups were assessed by the Pearson correlation test. Survival curves were plotted by the Kaplan-Meier method and the log-rank test was carried out to compare differences in survival. All statistical analyses were performed using the Prism 5.0 software (GraphPad 
Software Inc., USA). P $<0.05$ was considered to be statistically significant.

\section{Results}

\section{Elevated serum IL-6 is a useful predictor of recurrence after curative resection of HCC}

In this study, we compared the serum IL-6 concentration between two patient groups with or without recurrence after surgery of early stage HCC. As shown in Figure $1 \mathrm{~A}$, the concentrations of serum IL-6 determined prior to surgery in patients who developed recurrence were significantly higher than those of patients without recurrence during 5 years of follow-up $(21.45 \pm 6.98$ vs $16.09 \pm 5.87, P<0.05)$. We further assessed the correlation between serum IL-6 and survival time. As shown in Figure $1 \mathrm{~B}$, patients with a high serum IL-6
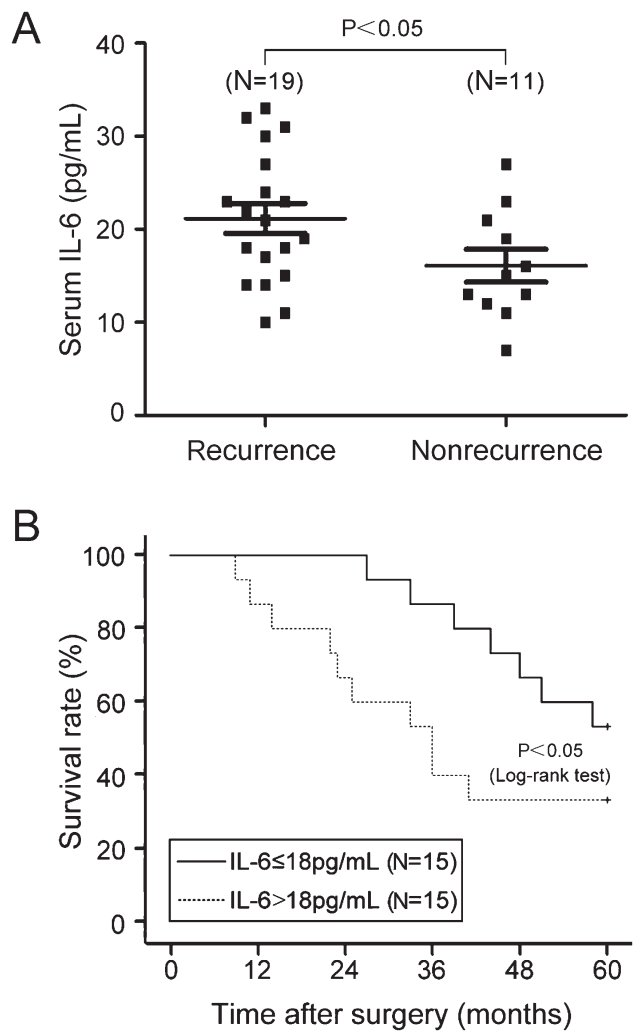

Figure 1. Serum IL-6 is a predictor of survival in hepatocellular carcinoma patients. $A$, Comparison of serum IL- 6 concentration with recurrence (least significant difference $t$-test). The number of patients is given in parentheses. Recurrence of hepatocellular carcinoma was based on image observations and the clinical course. $B$, Survival curve of patients who underwent curative resection of hepatocellular carcinoma. A median expression level was used as the cutoff. Kaplan-Meier analysis and the log-rank test showed that the survival rate of the group with serum IL-6 concentrations lower than $18 \mathrm{pg} / \mathrm{mL}$ was more favorable than that of the group with serum IL-6 higher than $18 \mathrm{pg} / \mathrm{mL}(P<0.05)$. concentration had significantly shorter survival times than those with a low serum IL-6 concentration (log-rank test, $P<0.05)$. These results indicate that a high serum IL-6 concentration is associated with postoperative recurrence and can be used as a predictor for survival of patients with $\mathrm{HCC}$.

\section{Serum IL-6 concentration is inversely correlated with miR-26a expression in HCC}

We next analyzed the association between miR-26a expression in HCC tissues and serum IL-6 concentration. A statistically significant inverse correlation was observed between IL-6 and miR-26a, high serum IL-6 concentration correlating with low expression of miR-26a in HCC tissues (Pearson correlation test, $r=-0.651, P<0.01$; Figure 2), whereas no correlation was detected between serum IL-6 concentration and miR-26a expression in adjacent noncancerous tissues $(r=-0.201, P>0.05)$.

\section{Incubation of HCC HepG2 cells with IL-6 suppresses} the expression of miR-26a via c-Myc activation

In this study, we hypothesized that elevated IL-6 expression contributes to the reduction of miR-26a expression in HCC through activation of the c-Myc pathway. We tested the effect of IL-6 stimulation on miR-26a expression in cultured HCC HepG2 cells. As previously reported, c-Myc protein was increased in a dose-dependent manner after 2-h incubation with IL-6 (Figure 3A). More importantly, the expression of miR-26a in HepG2 cells was decreased in response to incubation with IL-6 (Figure 3B). In order to further elucidate whether c-Myc was involved in the decreased expression of miR-26a upon IL-6 stimulation, a small molecule c-Myc inhibitor, 10058-F4, was used to examine the effect of c-Myc inhibition on IL-6-induced downregulation of

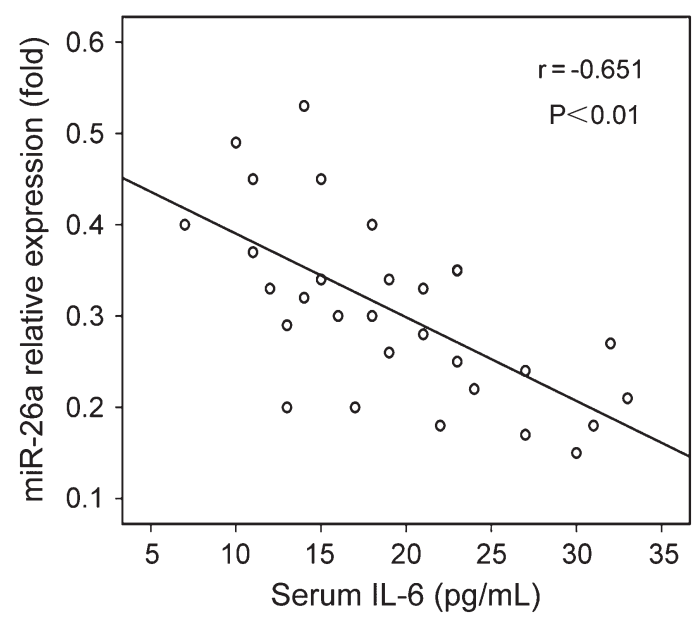

Figure 2. Correlation between serum IL-6 concentration and miR26 a expression in hepatocellular carcinoma. The Pearson correlation test showed that the correlation was $-0.651(P<0.01)$. 

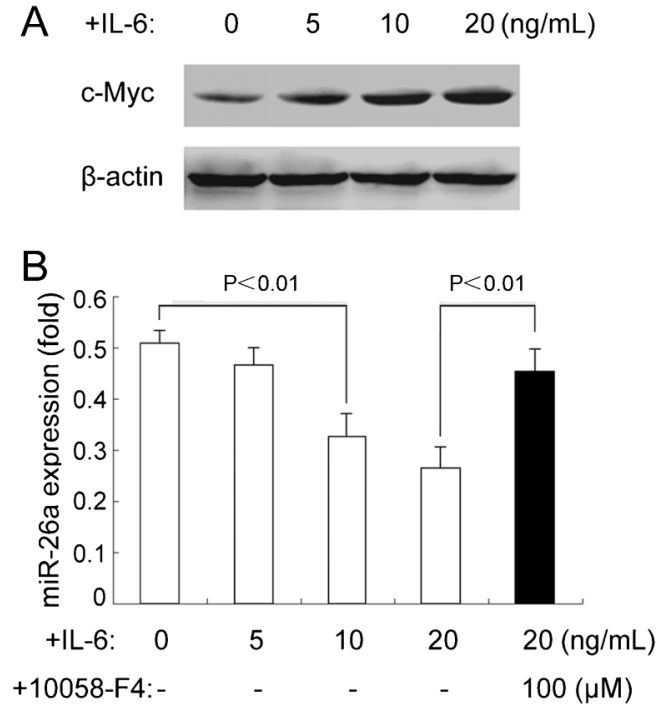

Figure 3. IL-6 stimulation leads to the reduction of miR-26a expression via activation of c-Myc in HepG2 cells. $A$, Representative immunoblots showed that c-Myc protein in HepG2 cells was increased in a dose-dependent manner upon IL-6 stimulation. $\beta$-actin was used as an internal control. $B$, Quantitative RT-PCR showed that miR-26a expression was reduced upon IL-6 stimulation, and the reduced miR-26a expression could be blocked by a c-Myc inhibitor (10058-F4). The data are representative of five independent experiments.

miR-26a. As expected, the c-Myc inhibitor blocked IL-6/ miR-26a signaling (Figure 3B). Taken together, these results support the view that IL-6 suppresses the expression of miR-26a via c-Myc activation in HCC cells.

\section{IL-6 incubation has no significant effect on the} expression of miR-26a in normal hepatocyte LO2 cells

The Pearson correlation test showed that there was no significant correlation between serum IL-6 concentration and miR-26a expression in adjacent noncancerous hepatic tissues. To verify this, we further analyzed the effect of IL-6 stimulation on the expression of miR-26a in normal human hepatocyte LO2 cells. As shown in Figure $4 \mathrm{~A}$, the expression of miR-26a in LO2 cells did not change significantly after incubation with IL-6. We also determined the protein concentration of c-Myc in LO2 cells after IL-6 incubation. The results of Western blot analysis showed that there were also no significant changes in c-Myc expression (Figure 4B), indicating that in normal hepatocyte cells IL-6 stimulation cannot activate c-Myc and thus suppress the expression of miR-26a. To determine whether or not c-Myc activation can lead to miR-26a reduction in normal hepatocyte cells, LO2 cells with increased c-Myc protein were selected (LO2-pc-cMyc cells, Figure 4C) and analyzed for miR-26a expression. Also, as shown in Figure 4D, no obvious miR-26 reduction was found in LO2 cells with upregulated c-Myc protein expression. These results suggest that the regulation of miR-26a expression in normal hepatocyte cells might be influenced by factors that differ from those affecting HCC cells.

\section{Discussion}

IL-6 has many physiologic roles and has been implicated in the process of tumorigenesis. A great variety of tumor types are stimulated by IL-6, including melanoma, Kaposi's sarcoma, lymphoma and leukemia, multiple myeloma, renal cell carcinoma, ovarian carcinoma, prostate carcinoma, colorectal carcinoma, and HCC (20). The ability of IL-6 to promote transcription of oncogene c-Myc in HCC HepG2 cells has been reported (9). Moreover, Chang et al. (3) recently demonstrated that hyperactivity of c-Myc leads to repression of miR-26a expression in various human cancer cells. Therefore, it is possible that elevated IL-6 expression increases the activity of c-Myc via paracrine or autocrine mechanisms and consequently leads to the reduction of miR-26a expression.

Several studies have revealed that serum IL-6 concentration is elevated in patients with $\mathrm{HCC}$ and that it can be used as a tumor marker, even more efficient than alpha-fetoprotein (AFP) (21-24). There are controversial reports about the significance of the use of serum IL-6 concentration for predicting survival. Giannitrapani et al. (21) reported that serum IL-6 has a significant positive correlation with the stage of HCC. Malaguarnera et al. (22) found that there is an elevated positive correlation between serum IL-6 and the size of HCC. Because the stage and size of tumors are both factors significantly associated with survival (25), it would be reasonable to propose that serum IL-6 concentration is also associated with survival in patients with HCC. However, Chau et al. (23) reported that there is no significant correlation between serum IL-6 and survival in HCC patients. It is interesting to note that the patients involved in the study of Chau et al. (23) were all from Chinese Taipei. Because most of the patients with HCC in China are HBV-related, and a large proportion of them have developed liver cirrhosis at the time of diagnosis, it might be difficult to evaluate the significance of serum IL-6 in predicting survival if cirrhosis-related death is not excluded (26-28). Gender is an important deciding factor for the expression of IL-6. Many studies have shown that estrogen inhibits IL-6 expression, which now is thought to be a key reason for the decreased susceptibility to HCC in females (29). Moreover, serum IL-6 concentration is affected by various clinical conditions, especially acute inflammation, which usually causes a significant increase in IL-6 expression (30). Due to the confounding factors discussed above, we did not find the predicted value of serum IL-6 in all of the 51 cases, which were initially included in this study (data not shown). However, after excluding female patients, 
A

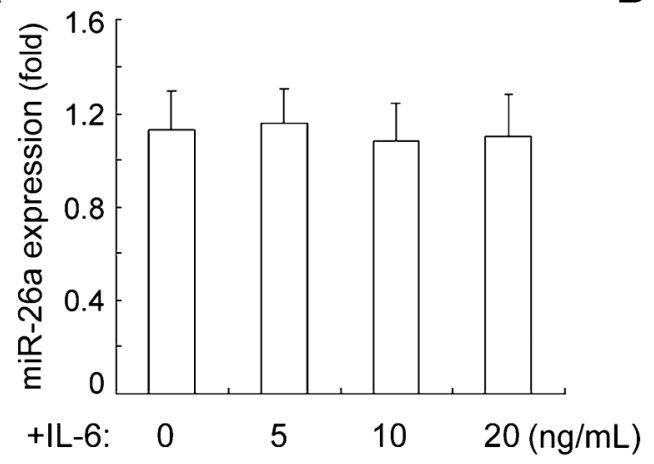

B

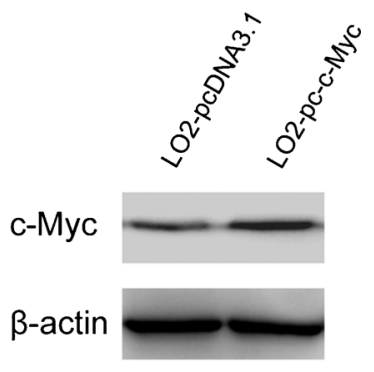

C

+IL-6: $\quad 0 \quad 5 \quad 10 \quad 20(\mathrm{ng} / \mathrm{mL})$

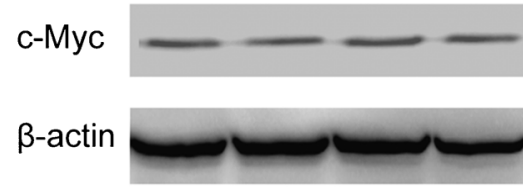

$\mathrm{D}$

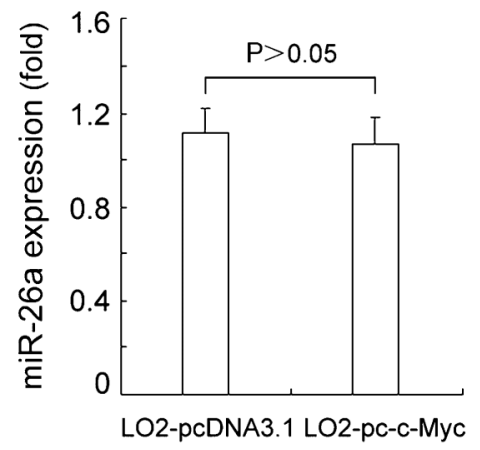

Figure 4. IL-6 stimulation does not affect the expression of miR-26a in LO2 cells. A, Quantitative RT-PCR showed that miR-26a expression in LO2 cells was not modified upon IL-6 stimulation ( $\mathrm{N}=5, \mathrm{P}>0.05)$. B, Expression of c-Myc protein in LO2-pc-c-Myc cells. $C$, Representative immunoblots also showed that c-Myc expression was not changed by IL-6. $D$, Expression of miR-26a in LO2pc-c-Myc cells. Compared with the expression of miR-26a in control LO2-pcDNA3.1 cells, the expression of miR-26a in LO2-pc-c-Myc cells was not significantly modified $(\mathrm{N}=5, \mathrm{P}>0.05)$.

patients with cirrhosis and acute infection from the statistical analysis, we observed that elevated serum IL-6 concentration can be considered to be a predictive risk factor for recurrence and reduced survival in our patients with HCC.

The oncogene c-Myc is pathologically activated in many human malignancies. Recent studies have revealed that activation of c-Myc may induce a widespread repression of miRNA expression (3). MiR-26a was shown to be repressed by c-Myc in almost all c-Myc-dependent tumors examined, thereby representing one of the most interesting c-Myc-repressed miRNAs $(3,5,6)$. Sander et al. (5) and Kota et al. (31) reported that the reduction of miR26a expression plays an important role in mediating c-Myc-dependent cell proliferation. The study of Kota et al. (31) in HepG2 cells also suggested that the reduction of miR-26a expression is involved in the apoptosis-resistant phenotype mediated by c-Myc. These observations indicated that miR-26a may be deeply involved in the transduction of the oncogenic c-Myc signal. Nevertheless, in our study, we also observed that in normal hepatocyte
LO2 cells IL-6 incubation cannot stimulate c-Myc activation and lead to miR-26a suppression. This is probably due to the fact that c-Myc expression is controlled by a large number of signaling pathways in normal cells (32), which therefore protect normal cells from c-Myc-mediated oncogenesis.

We demonstrated that IL-6 upregulation contributes to the reduction of miR-26a expression in $\mathrm{HCC}$ tissues. Because serum IL-6 concentration can be used as a valuable prognostic indicator for patients with $\mathrm{HCC}$, it may not be advisable to view miR-26a as an independent predictor of survival, and reduction of miR-26a expression should also be viewed as a poor prognostic marker.

\section{Acknowledgments}

Research supported by the National Foundation of Natural Sciences of China (\#81071727 and \#81101533), China Postdoctoral Science Foundation (\#20100481468 and \#201104755) and Natural Science Foundation of Hubei Province of China (\#2011CDB017). 


\section{References}

1. Calin GA, Croce CM. MicroRNA signatures in human cancers. Nat Rev Cancer 2006; 6: 857-866, doi: 10.1038/ nrc1997.

2. Iorio MV, Croce CM. MicroRNAs in cancer: small molecules with a huge impact. J Clin Oncol 2009; 27: 5848-5856, doi: 10.1200/JCO.2009.24.0317.

3. Chang TC, Yu D, Lee YS, Wentzel EA, Arking DE, West $\mathrm{KM}$, et al. Widespread microRNA repression by Myc contributes to tumorigenesis. Nat Genet 2008; 40: 43-50, doi: 10.1038/ng.2007.30.

4. Ji J, Shi J, Budhu A, Yu Z, Forgues M, Roessler S, et al. MicroRNA expression, survival, and response to interferon in liver cancer. N Engl J Med 2009; 361: 1437-1447, doi: 10.1056/NEJMoa0901282.

5. Sander S, Bullinger L, Klapproth K, Fiedler K, Kestler HA, Barth TF, et al. MYC stimulates EZH2 expression by repression of its negative regulator miR-26a. Blood 2008; 112: 4202-4212, doi: 10.1182/blood-2008-03-147645.

6. Mestdagh P, Fredlund E, Pattyn F, Schulte JH, Muth D, Vermeulen $\mathrm{J}$, et al. MYCN/C-MYC-induced microRNAs repress coding gene networks associated with poor outcome in MYCN/c-MYC-activated tumors. Oncogene 2010; 29: 1394-1404, doi: 10.1038/onc.2009.429.

7. Wen XY, Stewart AK, Sooknanan RR, Henderson G, Hawley TS, Reimold AM, et al. Identification of c-myc promoter-binding protein and $\mathrm{X}$-box binding protein 1 as interleukin- 6 target genes in human multiple myeloma cells. Int J Oncol 1999; 15: 173-178.

8. Shi Y, Frost PJ, Hoang BQ, Benavides A, Sharma S, Gera $\mathrm{JF}$, et al. IL-6-induced stimulation of c-myc translation in multiple myeloma cells is mediated by myc internal ribosome entry site function and the RNA-binding protein, hnRNP A1. Cancer Res 2008; 68: 10215-10222, doi: 10.1158/0008-5472.CAN-08-1066.

9. Barre B, Avril S, Coqueret O. Opposite regulation of myc and p21waf1 transcription by STAT3 proteins. J Biol Chem 2003; 278: 2990-2996, doi: 10.1074/jbc.M210422200.

10. Kishimoto T. Interleukin-6: from basic science to medicine 40 years in immunology. Annu Rev Immunol 2005; 23: 1-21, doi: 10.1146/annurev.immunol.23.021704.115806.

11. Nishimoto $\mathrm{N}$, Kishimoto $\mathrm{T}$. Interleukin 6 : from bench to bedside. Nat Clin Pract Rheumatol 2006; 2: 619-626, doi: 10.1038/ncprheum0338.

12. Hong DS, Angelo LS, Kurzrock R. Interleukin-6 and its receptor in cancer: implications for translational therapeutics. Cancer 2007; 110: 1911-1928, doi: 10.1002/cncr.22999.

13. Lukaszewicz M, Mroczko B, Szmitkowski M. [Clinical significance of interleukin-6 (IL-6) as a prognostic factor of cancer disease]. Pol Arch Med Wewn 2007; 117: 247-251.

14. Trikha M, Corringham R, Klein B, Rossi JF. Targeted antiinterleukin-6 monoclonal antibody therapy for cancer: a review of the rationale and clinical evidence. Clin Cancer Res 2003; 9: 4653-4665.

15. Knupfer H, Preiss R. Serum interleukin-6 levels in colorectal cancer patients - a summary of published results. Int $J$ Colorectal Dis 2010; 25: 135-140, doi: 10.1007/s00384-0090818-8.
16. Tas $F$, Oguz $H$, Argon A, Duranyildiz D, Camlica $H$, Yasasever $\mathrm{V}$, et al. The value of serum levels of IL-6, TNF-alpha, and erythropoietin in metastatic malignant melanoma: serum IL-6 level is a valuable prognostic factor at least as serum LDH in advanced melanoma. Med Oncol 2005; 22: 241-246, doi: 10.1385/ MO:22:3:241.

17. Knupfer H, Preiss R. Significance of interleukin-6 (IL-6) in breast cancer (review). Breast Cancer Res Treat 2007; 102: 129-135, doi: 10.1007/s10549-006-9328-3.

18. Chen C, Ridzon DA, Broomer AJ, Zhou Z, Lee DH, Nguyen JT, et al. Real-time quantification of microRNAs by stemloop RT-PCR. Nucleic Acids Res 2005; 33: e179, doi: 10.1093/nar/gni178.

19. Wang H, Hammoudeh DI, Follis AV, Reese BE, Lazo JS, Metallo SJ, et al. Improved low molecular weight Myc-Max inhibitors. Mol Cancer Ther 2007; 6: 2399-2408, doi: 10.1158/1535-7163.MCT-07-0005.

20. Liao WC, Lin JT, Wu CY, Huang SP, Lin MT, Wu AS, et al. Serum interleukin-6 level but not genotype predicts survival after resection in stages II and III gastric carcinoma. Clin Cancer Res 2008; 14: 428-434, doi: 10.1158/10780432.CCR-07-1032.

21. Giannitrapani L, Cervello M, Soresi M, Notarbartolo M, La RM, Virruso L, et al. Circulating IL-6 and sIL-6R in patients with hepatocellular carcinoma. Ann N Y Acad Sci 2002; 963: 46-52, doi: 10.1111/j.1749-6632.2002.tb04093.x.

22. Malaguarnera M, Di Fazio I, Laurino A, Romeo MA, Giugno I, Trovato BA. [Role of interleukin 6 in hepatocellular carcinoma]. Bull Cancer 1996; 83: 379-384.

23. Chau GY, Wu CW, Lui WY, Chang TJ, Kao HL, Wu LH, et al. Serum interleukin-10 but not interleukin-6 is related to clinical outcome in patients with resectable hepatocellular carcinoma. Ann Surg 2000; 231: 552-558, doi: 10.1097/ 00000658-200004000-00015.

24. Porta C, De Amici M, Quaglini S, Paglino C, Tagliani F, Boncimino A, et al. Circulating interleukin-6 as a tumor marker for hepatocellular carcinoma. Ann Oncol 2008; 19: 353-358, doi: 10.1093/annonc/mdm448.

25. Xu M, Bastos J, Dmitrewski J, Okajima H, Gunson B, Pirenne J, et al. Perihepatic packing in liver transplantation. Transplant Proc 1998; 30: 1850-1851, doi: 10.1016/S00411345(98)00454-0.

26. Liu J, Fan D. Hepatitis B in China. Lancet 2007; 369: 15821583, doi: 10.1016/S0140-6736(07)60723-5.

27. Chan HL, Sung JJ. Hepatocellular carcinoma and hepatitis B virus. Semin Liver Dis 2006; 26: 153-161, doi: 10.1055/s2006-939753.

28. Yamamoto J, Kosuge T, Saiura A, Sakamoto Y, Shimada K, Sano $T$, et al. Effectiveness of hepatic resection for earlystage hepatocellular carcinoma in cirrhotic patients: subgroup analysis according to Milan criteria. Jpn J Clin Oncol 2007; 37: 287-295, doi: 10.1093/jjco/hym025.

29. Tangkijvanich $P$, Mahachai $V$, Suwangool $P$, Poovorawan $Y$. Gender difference in clinicopathologic features and survival of patients with hepatocellular carcinoma. World $J$ Gastroenterol 2004; 10: 1547-1550. 
30. Nishimoto N. Interleukin-6 as a therapeutic target in candidate inflammatory diseases. Clin Pharmacol Ther 2010; 87: 483-487, doi: 10.1038/clpt.2009.313.

31. Kota J, Chivukula RR, O'Donnell KA, Wentzel EA, Montgomery CL, Hwang HW, et al. Therapeutic microRNA delivery suppresses tumorigenesis in a murine liver cancer model. Cell 2009; 137: 1005-1017, doi: 10.1016/ j.cell.2009.04.021.

32. Dang CV. MYC on the path to cancer. Cell 2012; 149: 2235, doi: 10.1016/j.cell.2012.03.003. 